\title{
Electron-scale nested quadrupole Hall field in Cluster observations of magnetic reconnection
}

\author{
N. Jain ${ }^{1}$ and A. S. Sharma ${ }^{2}$ \\ ${ }^{1}$ Max Planck Institute for Solar System Research, Justus-von-Liebig-Weg 3, Göttingen, Germany \\ ${ }^{2}$ Department of Astronomy, University of Maryland, College Park, MD 20742, USA \\ Correspondence to: N. Jain (jain@mps.mpg.de)
}

Received: 21 February 2015 - Revised: 7 May 2015 - Accepted: 8 May 2015 - Published: 12 June 2015

\begin{abstract}
This paper presents the first evidence of a new and unique feature of spontaneous reconnection at multiple sites in electron current sheet, viz. a "nested quadrupole" structure of the Hall field at electron scales, in Cluster observations. The new nested quadrupole is a consequence of electron-scale processes in reconnection. Whistler response of the upstream plasma to the interaction of electron flows from neighboring reconnection sites produces a large-scale quadrupole Hall field enclosing the quadrupole fields of the multiple sites, thus forming a nested structure. Electron-magnetohydrodynamic simulations of an electron current sheet yields a mechanism of the formation of a nested quadrupole.
\end{abstract}

Keywords. Magnetospheric physics (magnetotail; magnetic reconnection; numerical simulation studies)

\section{Introduction}

Magnetic reconnection is a fundamental process for the fast release of magnetic energy into kinetic and thermal energy in the laboratory, in space and in astrophysical plasmas. Collisionless reconnection develops in thin current sheets with thicknesses comparable to the electron skin depth $d_{\mathrm{e}}(=$ $\left.c / \omega_{\text {pe }}\right)$. The electron current sheet (ECS) with thickness $d_{\mathrm{e}}$ is embedded inside an ion current sheet with thickness $\sim d_{\mathrm{i}}\left(=c / \omega_{\mathrm{pi}}\right)$. The electron and ion dynamics are decoupled at this scale and the plasma is no longer frozen in the magnetic field, thus enabling reconnection. The Hall current due to the differential flow of ions and electrons in the reconnection region generates an out-of-plane magnetic field with a quadrupolar structure (Sonnerup, 1979; Mandt et al., 1994), which will be referred to as the Hall field. The quadrupole structure of the Hall field is an essential feature of collisionless reconnection and has been detected in space observations (Wygant et al., 2005; Borg et al., 2005; Asano et al., 2004), laboratory experiments (Ren et al., 2005) and simulations (Hesse et al., 2001).

The ECS is susceptible to secondary tearing instabilities which lead to the formation of magnetic islands due to spontaneous reconnection at multiple sites in ECS (Daughton et al., 2006). These secondary magnetic islands have been detected in Cluster observations of reconnection in Earth's magnetotail (Chen et al., 2008a; Wang et al., 2010). The interaction of neighboring sites in ECS leads to a new and unique feature, viz. a nested quadrupole structure of the Hall field (Jain and Sharma, 2009), unlike the single quadrupole in the case of reconnection at a single site. This feature arises in ECSs with a thickness $\left(\sim\right.$ a few $\left.d_{\mathrm{e}}\right)$ which is small compared to its extent ( $\sim$ a few $d_{i}$ ). Such current sheets are unstable to tearing instability, with a growth rate that has a maximum when the perturbation has a scale length of a few $d_{\text {e }}$ (Jain and Sharma, 2009; Attico et al., 2000), thus leading to reconnection at multiple sites. This paper presents the first evidence of a nested quadrupole structure of the Hall field in the Cluster observations of an electron-scale current sheet in Earth's magnetotail (Wygant et al., 2005). Electronmagnetohydrodynamic simulations of an ECS reveal the underlying physics of the formation of the nested structure.

The 1 October 2001 event observed by Cluster (Wygant et al., 2005) revealed many features of reconnection. While description of many of these features essentially requires kinetic models, e.g., electron pressure anisotropy in the inflow region (Chen et al., 2008a), many others can be described using simple fluid models. Here we show that Cluster observations shown in Fig. 2 can be appropriately de- 


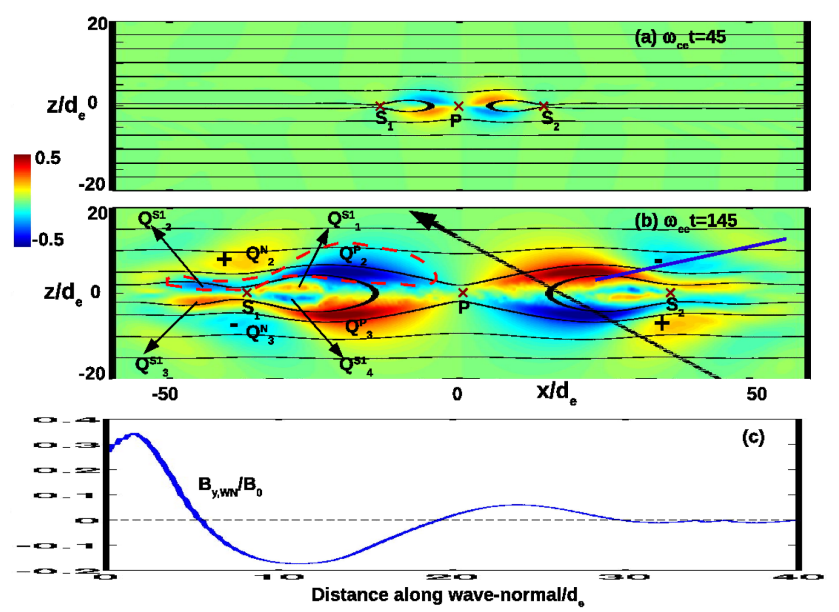

Figure 1. Magnetic field lines (black) plotted over color-coded $B_{y}$ at $\omega_{\text {ce }} t=45$ (a) and $=145$ (b). The primary $(P)$ and secondary $\left(S_{1}\right.$ and $\left.S_{2}\right)$ sites are marked by crosses $(\times S)$. At $\omega_{\text {ce }} t=145$, outward oblique propagation of whistlers from secondary sites forms a new quadrupole, marked with "+” and “-”. In (b), the poles of primary, secondary and new quadrupoles are marked by Q's (see text for definition) in the left half. The red dashed loop encloses a negative pole of the extended quadrupole. The blue line in the top right quadrant is at an angle of $19.5^{\circ}$ with the background magnetic field along $+x$ and approximates the wave normal. The profile of $B_{y}$ along the wave normal is shown in (c). The black line with an arrow in (b) shows a possible trajectory of the Cluster spacecraft.

scribed using an electron-magnetohydrodynamic (EMHD) model (Kingsep et al., 1990). In the observations, the typical gyro-radius for $100 \mathrm{eV}$ electrons is estimated to be $10 \mathrm{~km}$ $\left(\approx d_{\mathrm{e}} / 2\right)$ in the magnetic field of $3 \mathrm{nT}$. The half-thickness of the ECS in the observations is 3-5 electron inertial lengths, which is 6-10 times larger than the electron gyro-radius. Moreover, the magnitude of the magnetic field is never zero during the current sheet crossing (Fig. 3a in Wygant et al., 2005). The gyro-radius of $100 \mathrm{eV}$ electrons for the minimum magnitude of magnetic field $(\approx 1 \mathrm{nT})$ is $30 \mathrm{~km}$, which is approximately 2-3 times smaller than the half-thickness of the ECS. In the observations, ions are demagnetized and decoupled from electrons (Wygant et al., 2005). These considerations show that a fluid approximation at electron scales, viz. electron-magnetohydrodynamics, can be applied to describe the Cluster observations.

Although Cluster did not cross the $\mathrm{X}$ point, kinetic processes near the $\mathrm{X}$ point, e.g., meandering orbits of electrons, or near the weak field regions on the spacecraft trajectory may influence the dynamics. However the excellent agreement of EMHD simulations with the observations, as we show, indicates that the essential physics are captured by EMHD model.

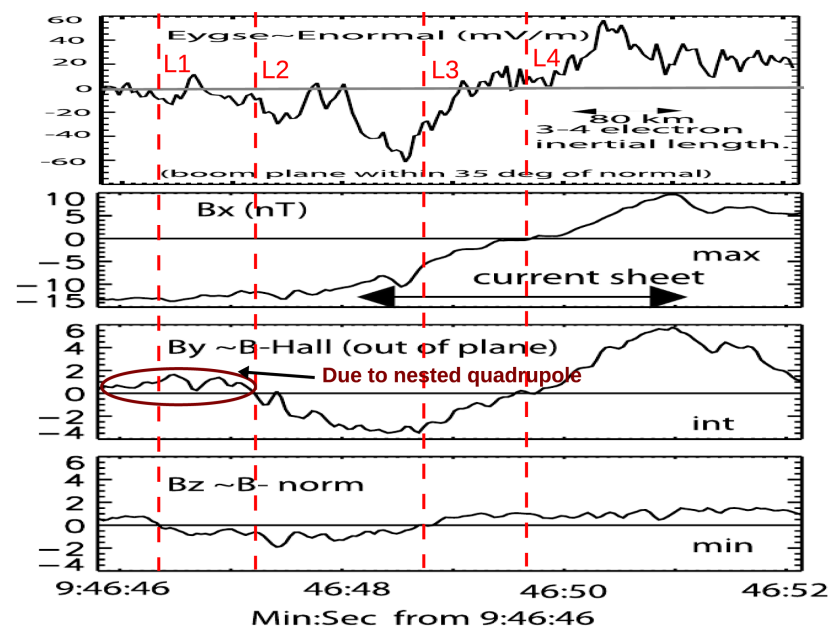

Figure 2. Observation of electron-scale current sheet by Cluster (adopted from Fig. 3a of Wygant et al., 2005). Top panel: $E_{y \mathrm{GSE}}$ ( $y$ component of electric field in GSE coordinate system). Three bottom panels: $x, y$ and $z$ components of magnetic field in boundary normal coordinate system. Vertical dashed lines $\left(L_{1}-L_{4}\right)$ mark the zero crossings of the magnetic field components.

\section{Nested quadrupole structure of the Hall field}

Spontaneous reconnection at multiple sites in an ECS developing into primary and secondary sites (Jain and Sharma, 2009), shown in Fig. 1, is modeled using EMHD simulations. Here length is normalized by $d_{\mathrm{e}}$, magnetic field by the asymptotic value $B_{0}$, and time by $\omega_{\mathrm{ce}}^{-1}=\left(e B_{0} / m_{\mathrm{e}}\right)^{-1}$. Fig. 1a shows the structure of the Hall field $B_{y}$ in the early stage $\left(\omega_{\mathrm{ce}} t=45\right)$, which evolves into the late stage $\left(\omega_{\mathrm{ce}} t=\right.$ $145)$, shown in Fig. 1b. At $\omega_{\mathrm{ce}} t=45$ the reconnection is dominant at the primary site $(P)$ in the center of the simulation domain $(x=z=0)$. The field lines reconnected at the primary site $P$ reconnect again at the secondary sites $\left(S_{1}\right.$ at $x \approx-16 d_{\mathrm{e}}$ and $S_{2}$ at $x \approx 16 d_{\mathrm{e}}$ ), giving rise to reconnection at multiple sites. The quadrupole structure of the out-ofplane magnetic field is clearly developed around the primary site, while it is not yet recognizable at the secondary sites. At $\omega_{\text {ce }} t=145$, the central site remains dominant and the secondary sites are pushed away by the outflows from the central site. We label the quadrupole Hall fields associated with $S_{1}, S_{2}$ and $P$ as $Q^{S_{1}}, Q^{S_{2}}$ and $Q^{P}$, respectively. The new quadrupole, marked as $Q^{N}$, forms due to the interactions of the inflow to the secondary sites and the outflow from the primary site (Jain and Sharma, 2009). The poles of a quadrupole are numbered counterclockwise beginning with 1 for the top right pole to 4 for the bottom right pole. An individual pole of a quadrupole is represented by a subscript to $Q$ 's. For example, $Q_{1}^{S_{1}}$ represents the top right pole of the quadrupole associated with the reconnection site $S_{1}$. The poles of the primary, secondary and new quadrupoles are marked only in the left half of Fig. $1 \mathrm{~b}$. The poles $Q_{1}^{S_{1}}$ and $Q_{4}^{S_{1}}$ of the secondary 
quadrupole at $S_{1}$ penetrate between the poles $Q_{2}^{P}$ and $Q_{3}^{P}$ of the primary quadrupole. At the same time, the poles $Q_{2}^{S_{1}}$ and $Q_{3}^{S_{1}}$ of the secondary quadrupole at $S_{1}$ connect to the poles $Q_{2}^{P}$ and $Q_{3}^{P}$ of the primary quadrupole, respectively, thus increasing the extent of the primary quadrupole $Q^{P}$. One of the negative poles $\left(Q_{2}^{P}+Q_{2}^{S_{1}}\right)$ of the extended quadrupole is enclosed by a closed loop (red dashed line) in Fig. 1b. The extended quadrupole is nested inside the new quadrupole $\left(Q^{N}\right)$, the poles of which are also marked ("+" and "-") in Fig. 1b.

A striking feature of spontaneous reconnection at multiple sites is the new quadrupole, which, unlike the other three quadrupoles in Fig. 1b, is not directly associated with a reconnection site but arises from their interaction. The physics of the new quadrupole are the whistler response of the upstream plasma to the interaction of inflow to the secondary (weak) sites and outflow from the primary (dominant) site (Jain and Sharma, 2009). Because of the magnetic field structure of reconnection, the whistler perturbations are anchored in phase at their origin and propagate away from the reconnection region. The direction of propagation is very well approximated by the wave normal (shown by the blue line in Fig. 1b), which is at a Storey angle of $19.5^{\circ}$ (Storey, 1953; Singh, 2011) with the background magnetic field along $x$. Figure 1c shows the out-of-plane magnetic field $B_{y}$,WN along the wave normal. The wave propagates away from the reconnection region while its amplitude diminishes. The distance between positive and negative peaks is $\approx 12 d_{\mathrm{e}}$, giving a wave number $k d_{\mathrm{e}} \approx 0.25$, as expected for frequency $\omega=0.1 \omega_{\mathrm{ce}}$ (Singh, 2007). The extension of the primary quadrupole along $x$ and the formation of a new quadrupole due to the whistler perturbation at secondary sites in the manner described above make the overall structure a nested structure of quadrupoles.

The EMHD simulations are of the early phase of reconnection, in the sense that the results are valid for a short time (less than the ion cyclotron time) after the development of the instability in the ECS. However the results are in general valid for the later stage as well because the spatial structures arise mainly from the whistler eigenmode, which will persist with modifications due to coupling to ions, other modes and inhomogeneities.

\section{Cluster observations of nested quadrupole}

The Cluster spacecraft crossed the reconnection region at distances of $\sim 18 R_{\mathrm{E}}$ in Earth's magnetotail on 1 October 2001. Among the four spacecraft, SC4 was closest to the X line and crossed the current sheet on the earthward side between 09:46:48 and 09:46:51 UT, and the profiles of electric and magnetic field are shown in Fig. 2 (Fig. 3 in Wygant et al., 2005). The change in sign of the magnetic field components is critical to the structure of the Hall field, and the time marks for these are shown by the vertical dashed lines in Fig. 2, viz. $L_{1}$ for $B_{z}, L_{2}$ for $B_{y}, L_{3}$ for $B_{z}$, and $L_{4}$ for $B_{x}$ and $B_{y}$.

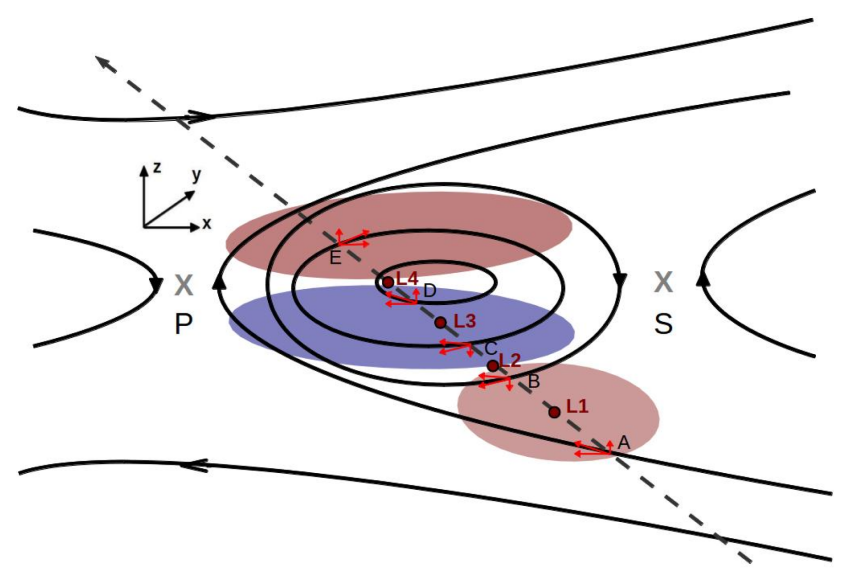

Figure 3. A schematic of reconnection at a primary $(P)$ and a secondary $(S)$ sites. Components of the magnetic field in the $x$ and $z$ directions are shown by red arrows at select locations (A, B, C, $\mathrm{D}$ and $\mathrm{E}$ ) on a spacecraft trajectory (black dashed line). The sign of the out-of-plane magnetic field $B_{y}$ in the regions of interest is represented by color-shaded (positive by reddish and negative by blueish) regions. The locations of zero crossings of the magnetic field components, marked by lines $L_{1}-L_{4}$ in Figs. 2 and 4, are approximately indicated on the trajectory by filled circles.

A schematic of the magnetic field structure corresponding to the Cluster observations (Fig. 2) is shown in Fig. 3, and consists of a primary site, with $\mathrm{X}$ point at $P$, and a secondary site with $\mathrm{X}$ point at $S$. In the standard picture of 2-D reconnection with a single reconnection site, i.e., in the absence of the secondary sites, $B_{z}$ should have the same sign on any one side (tailward or earthward) of the $y-z$ plane containing the $\mathrm{X}$ point $P$, and change sign only when spacecraft crosses this plane. But for such a passage by a spacecraft, the peak of the out-of-plane Hall field $\left(B_{y}\right)$ should not coincide with the zero crossing of the normal magnetic field $\left(B_{z}\right)$. This is because the peaks of the Hall field are located away from this plane. Thus the change in sign of $B_{z}$ at $L_{3}$ coinciding with the peak of the Hall field is not consistent with reconnection at a single site. The change in sign of $B_{z}$ without crossing an $\mathrm{X}$ point is possible, however, when a spacecraft crosses the current sheet between a primary $(P)$ and a secondary $(S)$ reconnection site, e.g., along the dashed line in Fig. 3. The simultaneous occurrence of the peak of $B_{y}$ and zero crossing of $B_{z}$, i.e., bipolar $B_{z}$ and unipolar or doublepeaked $B_{y}$, is a generic feature used to identify a magnetic island between two reconnection sites (Chen et al., 2008b). Although the presence of a magnetic island between the two reconnection sites, $P$ and $S$, is enough for the sign reversal of $B_{z}$ at $L_{3}$ and $B_{y}$ at $L_{4}$, the small positive $B_{z}$ on the left of $L_{1}$ and $B_{y}$ on the left of $L_{2}$ additionally requires the weak or secondary site to be inside the region created by the dominant or primary site. The spacecraft first encounters magnetic field lines (at A with $B_{x}<0$ and $B_{y}, B_{z}>0$ ) reconnected at the primary site $P$ but not reconnected at the secondary site 


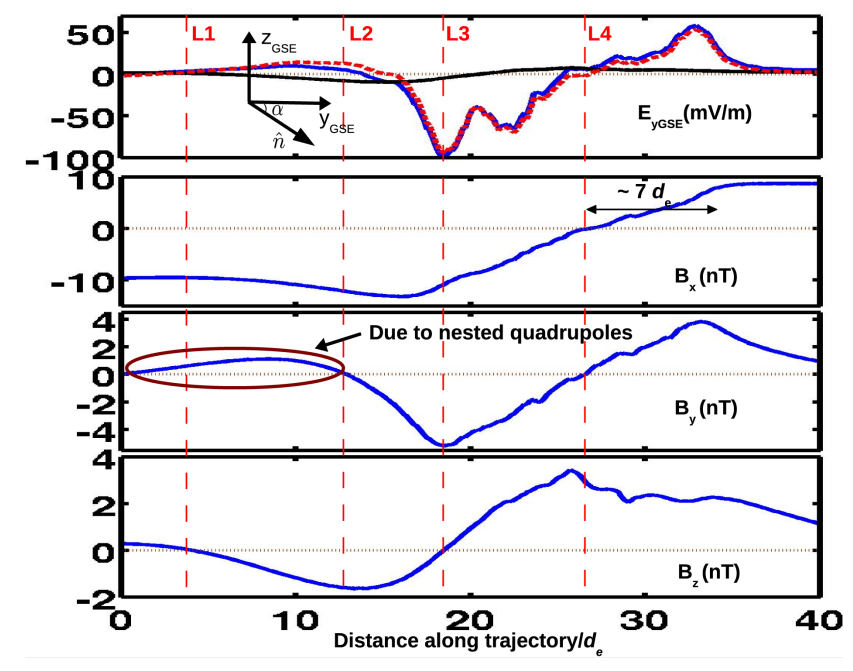

Figure 4. Simulated electric and magnetic field profiles along the trajectory shown in Fig. 1b. Top panel: the $y$ component of the electric field ( $E_{y \mathrm{GSE}}$, blue) transformed from the simulation or boundary normal to GSE coordinate system, the normal component (red) and current-aligned (black) electric field of $E_{y \mathrm{GSE}}$. Also shown is the boundary normal vector in the $y_{\mathrm{GSE}}-z_{\mathrm{GSE}}$ plane. Three bottom panels: the $x, y$ and $z$ components of the magnetic field in boundary normal coordinate system. Vertical dashed lines $\left(L_{1}-L_{4}\right)$ mark the zero crossings of the magnetic field components.

$S$ (see Fig. 3). It then encounters the field lines of the magnetic islands formed due to the reconnection both at $P$ and $S$, first in the region below the plane containing the primary and secondary sites, viz. the south lobe (at points B, C and $\mathrm{D}$ ), and then in the north lobe (at point E). Since $B_{z}$ changes sign from positive at $\mathrm{A}$ to negative at $\mathrm{B}$ while $B_{x}<0$ and $B_{y}>0$ both at $\mathrm{A}$ and $\mathrm{B}$, the first zero crossing of $B_{z}$ (marked by line L1 in Fig. 2) must be somewhere between A and B as shown in Fig. 3. By looking at the signs of the magnetic field components, the approximate locations of their other zero crossings, marked by lines L2, L3 and L4 in Fig. 2, can be identified on the virtual spacecraft trajectory as shown in Fig. 3.

For comparison with Cluster observations, Fig. 4 shows the simulation profiles of electric and magnetic fields (in unnormalized units using $B_{0}=10 \mathrm{nT}$ and $d_{\mathrm{e}}=20 \mathrm{~km}$ for Cluster observations) along the trajectory shown in Fig. 1b, as functions of distance along the trajectory. Similar to Fig. 2, the vertical dashed lines in this figure mark the zero crossing of $B_{x}$ at $L_{4}, B_{y}$ at $L_{2}$ and $L_{4}$, and $B_{z}$ at $L_{1}$ and $L_{3}$. The profiles of the $y$ component of electric field and all components of magnetic field in Fig. 2 are in the geocentric solar ecliptic (GSE) and boundary normal coordinate systems, respectively. In the boundary normal coordinate system, $z$ is normal to the current sheet surface, $y$ is along the direction of current and $(x, y, z)$ forms a right-handed coordinate system. Since the simulations are in boundary normal coordinate system, the profile of the electric field in Fig. 4 is obtained by transforming it from boundary normal to the GSE system. The boundary normal vector $\hat{n}=-0.05 \hat{x}_{\mathrm{GSE}}+0.80 \hat{y}_{\mathrm{GSE}}-$ $0.59 \hat{z}_{\mathrm{GSE}}$ of the highly tilted current sheet in Cluster observations is almost in the $y_{\mathrm{GSE}}-z_{\mathrm{GSE}}$ plane and shown in the top panel of Fig. 4. Assuming the current sheet in the simulations to have the same orientation with respect to the GSE coordinate system, the $y$ component of the electric field in the latter can be obtained from $E_{y \mathrm{GSE}}=E_{y} \sin (\alpha)-E_{z} \cos (\alpha)$, where $\alpha$ is the angle between the normal vector and the $\hat{y}_{\mathrm{GSE}}$, with $\cos (\alpha)=0.8$.

The electric and magnetic field profiles in the Cluster observation (Fig. 2) and EMHD simulation (Fig. 4) are remarkably similar not only in magnitude but also in the scale and pattern of variation. The current sheet crossing, represented by the change in $B_{x}$ from $\approx-10$ to $\approx 10 \mathrm{nT}$ in observations (during $\sim 46: 48-46: 51$, Fig. 2) and simulations (Fig. 4), provides more details on the reconnection in the magnetotail. The half-thickness of the current sheet in simulations $\approx 7 d_{\mathrm{e}}$ compares well with the observed values $\sim 3-5 d_{\mathrm{e}}$. The steplike structures of $B_{x}$ inside the current sheet are present both in simulations and observations, and indicate a filamentary structure in the current sheet.

Associated with the current sheet crossing, $E_{y \mathrm{GSE}}$ and Hall field $B_{y}$ have bipolar forms that change their signs from negative to positive. The positive and negative peaks of the bipolar structures of $E_{y \mathrm{GSE}}$ and $B_{y}$ in observations and simulations are very similar. Consistent with the observations, Fig. 4 shows that $E_{y \mathrm{GSE}}$, given by $E_{y} \sin (\alpha)-E_{z} \cos (\alpha)$, is dominated by the normal component of the electric field, $E_{z} \cos (\alpha)$, due to the tilt of the current sheet with respect to the GSE coordinate system.

The normal component of magnetic field $B_{z}$ remains positive during the current sheet crossing but is negative just before the current sheet crossing (between $L_{1}$ and $L_{3}$ ). The zero crossing of $B_{z}$ at $L_{3}$ coincides with the edge of the current sheet and negative peaks of $E_{y \mathrm{GSE}}$ and $B_{y}$. Both $B_{z}$ and $B_{y}$ have positive values before their first zero crossings at $L_{1}$ and $L_{2}$, respectively. In the simulations, the positive $B_{y}$ on the left of $L_{2}$ is due to the crossing of a positive pole (marked by "+" on the positive $x$ side in Fig. 1b) of the new (outer) quadrupole structure of $B_{y}$. The positive $B_{y}$ on the left of $L_{2}$ in Fig. 2 can be identified with the new quadrupole, and the Cluster observation is consistent with a nested quadrupole structure of the out-of-plane magnetic field. Note that the peak value of $B_{y}$ on the left of $L_{2}$ is $\approx 30 \%$ of the largest peak of the observed $B_{y}$ and is thus significant and due to the physical electron-scale processes.

It should be noted that the Cluster observations (Wygant et al., 2005) have uncertainties arising from the high variability in the plasma in the reconnection region, the deviation from two-dimensionality and the validity of the minimum variance analysis. The uncertainties associated with the coordinate system can be as much as $20^{\circ}$, and the normal component of the magnetic field, $B_{z}$, is $\sim 1 \mathrm{nT}$. Thus the interpretation presented here is subject to such uncertainties. 
With higher resolution measurements of the fields, e.g., from NASA/MMS mission, these issues are likely to be resolved. Also, the electron measurements could be used to compare with the flows seen in the simulations to complement the magnetic field data.

\section{Discussion and conclusions}

The formation of the nested structure of quadrupoles of the Hall magnetic field requires not only the presence of multiple sites but also the dominance of one site over the neighboring sites. Simulations with three reconnection sites of equal strength (excited by initializing the simulations with a single wavelength perturbation with three wavelengths fitting in the length of the simulation box along $x$ ) show that the out-ofplane magnetic field does not develop a nested structure of quadrupoles. Although the quadrupole structure of $B_{y}$ forms at each reconnection site, the inflow to one site and outflow from the neighboring site do not interact in the manner that results into the nested quadrupole structure. In natural situations, e.g., in the magnetotail, reconnection at multiple sites is expected, with the one initiated first being dominant over the adjacent sites. Further, in the magnetotail, the monotonic decrease in the magnetic field away from Earth (along $x$ ) will introduce asymmetry among the multiple reconnection sites, thus leading to the nested Hall field.

In the Cluster observations, the total time of crossing $(\approx 6 \mathrm{~s})$ is close to the ion cyclotron period and thus captured the electron-dominated physics of reconnection. Since these electron-scale observations are by a single spacecraft when the other three spacecrafts were separated by distances much larger than typical electron scales $(\sim 20 \mathrm{~km})$, the spatial and temporal variations are not uniquely distinguished. However, the EMHD simulations show that the electronscale structures form very quickly, in a time of the order of tens of electron cyclotron periods, but evolve very slowly after their formation (Jain and Sharma, 2009). Thus the structures observed by Cluster are consistent with spatial variations as described above. The forthcoming multi-spacecraft NASA/MMS mission, designed to resolve the electron scales in the magnetosphere and to distinguish between spatial and temporal variations, will provide key details of the spatiotemporal structure.

In conclusion, the nested quadrupole structure of the Hall magnetic field is identified in Cluster observations and the underlying mechanism is revealed by EMHD simulations of an ECS. Many details of the electron-scale physics and the connection to the larger-scale ion processes remain yet unexplored. Such studies will require new studies of electronscale physics in simulations, experiments and satellite observations of magnetic reconnection. In particular, the results presented in this paper provide a critical step for a deeper understanding of reconnection at electron scales using new kinetic simulations that resolve the electron scales clearly and the data for electron-scale physics from the upcoming NASA/MMS mission.

Acknowledgements. This research was supported by NSF grant AGS-1027185.

The article processing charges for this open-access publication were covered by the Max Planck Society.

The topical editor E. Roussos thanks one anonymous referee for help in evaluating this paper.

\section{References}

Asano, Y., Mukai, T., Hoshino, M., Saito, Y., Hayakawa, H., and Nagai, T.: Current sheet structure around the near-Earth neutral line observed by Geotail, J. Geophys. Res., 109, A02212, doi:10.1029/2003JA010114, 2004.

Attico, N., Califano, F., and Pegoraro, F.: Fast collisionless reconnection in the whistler frequency range, Phys. Plasmas, 7, 2381, doi:10.1063/1.874076, 2000.

Borg, A. L., Oieroset, M., Phan, T. D., Mozer, F. S., Pedersen, A., and Mouikis, C.: Cluster encounter of a magnetic reconnection diffusion region in the near-Earth magnetotail on September 19, 2003, Geophys. Res. Lett., 12, L19105, doi:10.1029/2005GL023794, 2005.

Chen, L. J., Bessho, N., Lefebvre, B., Vaith, H., Fazakerley, A., Bhattacharjee, A., Puhl-Quinn, P. A., Runov, A., Khotyaintsev, Y., Vaivads, A., Georgescu, E., and Torbert, R.: Evidence of an extended electron current sheet and its neighboring magnetic island during magnetotail reconnection, J. Geophys. Res., 113, A12213, doi:10.1029/2008JA013385, 2008a.

Chen, L. J., Bhattacharjee, A., Puhl-Quinn, P. A., Yang, H., Bessho, N., Imada, S., Muhlbachler, S., Daly, P. W., Lefebvre, B., Khotyaintsev, Y., Vaivads, A., Fazakerley, A., and Georgescu, E.: Observation of energetic electrons within magnetic islands, Nature Phys., 4, 19-23, doi:10.1038/nphys777, 2008b.

Daughton, W., Scudder, J., and Karimabadi, H.: Fully kinetic simulations of undriven magnetic reconnection with open boundary conditions, Phys. Plasmas, 13, 072101, doi:10.1063/1.2218817, 2006.

Hesse, M., Birn, J., and Kuznetsova, M.: Collisionless magnetic reconnection: Electron processes and transport modelling, J. Geophys. Res., 106, 3721, doi:10.1029/1999JA001002, 2001.

Jain, N. and Sharma, A. S.: Electron Scale Structures in Collisionless Magnetic Reconnection, Phys. Plasmas, 16, 050704, doi:10.1063/1.3134045, 2009.

Kingsep, A. S., Chukbar, K. V., and Yan'kov, V. V.: Reviews of Plasma Physics, vol. 16, Consultants Bureau, New York, USA, p. 243, 1990.

Mandt, M. E., Denton, R. E., and Drake, J. F.: Transition to Whistler Mediated Magnetic Reconnection, Geophys. Res. Lett., 21, 7376, doi:10.1029/93GL03382, 1994.

Ren, Y., Yamada, M., Gerhardt, S., Ji, H., Kulsrud, R., and Kuritsyn, A.: Experimental verification of the Hall effect during magnetic reconnection in a laboratory plasma, Phys. Rev. Lett., 95, 055003, doi:10.1103/PhysRevLett.95.055003, 2005. 
Singh, N.: Group velocity cones in diverging magnetic reconnection structures, J. Geophys. Res., 112, A07209, doi:10.1029/2006JA012219, 2007.

Singh, N.: Whistler mode based explanation for the fast reconnection rate measured in the MIT Versatile Toroidal Facility, Phys. Rev. Lett., 107, 245003, doi:10.1103/PhysRevLett.107.245003, 2011.

Sonnerup, B. U. O.: in Solar System Plasma Physics, vol. 3, NorthHolland, Amsterdam, the Netherlands, 1979.

Storey, L. R. O.: An investigation of whistling atmospherics, Phil. Trans. Roy. Soc. Ser. A, 246, 113-141, doi:10.1098/rsta.1953.0011, 1953.
Wang, R., Lu, Q., Du, A., and Wang, S.: In Situ observations of a secondary magnetic island in an ion diffusion region and associted energetic electrons, Phys. Rev. Lett., 104, 175003, doi:10.1103/PhysRevLett.104.175003, 2010.

Wygant, J. R., Cattell, C. A., Lysak, R., Song, Y., Dombeck, J., McFadden, J., Mozer, F. S., Carlson, C. W., Parks, G., Lucek, E. A., Balogh, A., Andre, M., Reme, H., Hesse, M., and Mouikis, C.: Cluster observations of an intense normal component of the electric field at a thin reconnecting current sheet in the tail and its role in the shock-like acceleration of the ion fluid into the separatrix region, J. Geophys. Res., 110, A09206, doi:10.1029/2004JA010708, 2005. 